\title{
Produção e Qualidade de Melões Sob Diferentes Arranjos do Sistema de Irrigação e Coberturas do Solo
}

\author{
Wiltemberg de Brito Pereira ${ }^{1}$, Carlos Eduardo Franco Possídio ${ }^{1}$, \\ José Sebastião Costa de Sousa $^{1}$ (D), Welson Lima Simões ${ }^{2}$ D, Caio Márcio Guimarães Santos ${ }^{1}$ \\ ${ }^{1}$ Instituto Federal de Educação, Ciência e Tecnologia do Sertão Pernambucano, Campus \\ Petrolina Zona Rural, Petrolina, PE, Brasil. \\ ${ }^{2}$ Embrapa Semiárido, Petrolina, PE, Brasil.
}

Recebido em: 7 de Agosto de 2020 - Aceito em: 6 de Novembro de 2020

\begin{abstract}
Resumo
Objetivou-se com este trabalho avaliar aspectos produtivos e de qualidade de meloeiros (Cucumis melo L.) sob diferentes formas de cultivo em Petrolina/PE durante dois ciclos produtivos. Adotou-se delineamento experimental de blocos casualizados com parcelas subsubdivididas em esquema fatorial $2 \times 2 \times 3$, sendo, dois híbridos de melão amarelo (Gladial e SF 10/00 F1) nas parcelas, dois arranjos do sistema de irrigação (uma e duas fileiras de gotejadores por fileira de plantas) nas subparcelas, e três tipos de coberturas do solo (mulchings preto e cinza e sem cobertura) nas subsubparcelas, com seis repetições. Foram avaliados, comprimento, largura e número total e comercial de frutos, produtividade, uso eficiente da água, sólidos solúveis totais, firmeza e acidez da polpa. Os maiores índices de produtividade, uso eficiente da água e número de frutos comerciais foram obtidos com o uso de mulching (independente da cor) com uma fileira de gotejadores por fileira de plantas. Para os índices pós-colheita os tratamentos não diferiram entre si.
\end{abstract}

Palavras-chave Cucumis melo, mulching, uso eficiente da água.

\section{Production and Quality of Melons Under Different Arrangements of the Irrigation System and Ground Cover}

\begin{abstract}
The aim of this work was to evaluate productive and quality aspects of melons (Cucumis Melo L.) under different forms of cultivation in Petrolina/PE during two cycles of production. A randomized block design was adopted with sub-divided plots in a $2 \times 2 \times 3$ factorial scheme, with two yellow melon hybrids (Gladial and SF 10/00 F1) in the plots, two irrigation system arrangements (one and two rows drippers per row of plants) in the subplots, and three types of soil cover (black and gray mulching and without cover) in the subplots, with six replications. Were evaluated length, width, total and commercial number of fruits, productivity, efficient use of water, total soluble solids, firmness and acidity of the pulp were evaluated. The highest rates of productivity, efficient use of water and number of commercial fruits were obtained with the use of mulching (regardless of color) with a row of drippers per row of plants. For post-harvest rates, the treatments did not differ.
\end{abstract}

Keywords Cucumis melo, mulching, efficient water use.

\section{Introdução}

O semiárido brasileiro por apresentar condições edafoclimáticas propícias ao cultivo do meloeiro, Cucumis Melo L., garante anualmente cerca de $95 \%$ das exportações da cultura, com destaque aos estados do Rio Grande do Norte, Ceará, Bahia e Pernambuco. Nestes últimos o polo produtivo Petrolina-PE/Juazeiro-BA, e circunvizinhanças, responde por mais de um oitavo da área cultivada com melão no país (Barros et al., 2019; Pinto et al., 2019).

Autor para correspondência: José Sebastião Costa de Sousa, sebastiao.costa@ifsertao-pe.edu.br. 
As cultivares de melão de maior expressão nacional são do tipo Pele de Sapo, Amarelo, Cantaloupe e Gália, com tendência crescente a exploração de híbridos pelo seu potencial produtivo, especialmente quando irrigado (Lima, 2015; Cavalcante Neto et al., 2020). Irrigação esta que é promovida predominantemente pelo sistema de gotejamento, por evitar molhar a parte aérea da planta, permitir maior controle na quimigação e maior eficiência de uso da água. Comumente estes sistemas são munidos de emissores espaçados de 0,30 a $0,50 \mathrm{~m}$ entre plantas e 2,00 m entre fileiras (Cavalcanti et al., 2008; Batista et al., 2009; Pereira et al., 2017; Guimarães et al., 2020). Gerando áreas molhadas na ordem de $25 \%$ (para instalações convencionais de uma fileira de gotejadores por fileira de plantas), valor inferior ao recomendado pela literatura, que é de 33\% para regiões áridas e semiáridas (Vermeiren e Jobling, 1997; Bernardo et al., 2019). Cabendo-se, portanto, entre outros fatores, a investigação da resposta da cultura a outros arranjos do sistema de irrigação, que propiciem maior área molhada.

Outra importante técnica geradora de benefícios para o meloeiro é a cobertura do solo. Medeiros et al. (2006) e Silva et al. (2016), por exemplo, encontraram produtividades até 39\% maiores para o cultivo de híbridos Cantaloupe e Amarelo sob mulching em comparação aos cultivos descobertos. Já Ibarra et al. (2001) e Braga et al. (2017) identificaram influência nos rendimentos e nos aspectos vegetativos de melões, até pela cor do mulching usado.

Contudo, tais informações ainda são escassas para os melões 'Gladial' e 'SF 10/00'. Desta forma, objetivou-se com este trabalho avaliar atributos produtivos e de póscolheita em híbridos de melão amarelo, 'Gladial' e 'SF 10/00 F1', submetidos a diferentes arranjos do sistema de irrigação e coberturas do solo.

\section{Material e Métodos}

O experimento foi conduzido, no período compreendido entre 09 de abril a 08 de junho, primeiro ciclo, e de 30 de agosto a 08 de novembro de 2016, segundo ciclo, no Campus Petrolina Zona Rural do IF Sertão-PE, em Petrolina-PE $\left(9^{\circ} 20^{\prime} 13^{\prime \prime}\right.$ Sul, $40^{\circ} 42^{\prime} 01^{\prime \prime}$ Oeste, altitude de $413 \mathrm{~m}$ ). Região que apresenta classificação climática de Köppen do tipo BSh, ou seja, clima semiárido quente com chuvas predominantes no verão (Azevedo et al., 2003).
O solo da área experimental foi classificado como Argissolo Amarelo (Embrapa, 2006), com características físico-químicas da Tabela 1.

O delineamento experimental adotado foi o de blocos casualizados com parcelas subsubdivididas em esquema fatorial misto $2 \times 2 \times 3$, sendo, dois híbridos de melão amarelo (H1 - melão 'Gladial' e, H2 - melão 'SF 10/00 F1') nas parcelas; dois arranjos do sistema de irrigação (S1 - uma e S2 - duas, fileiras de gotejadores por fileira de plantas, representando 25 e $50 \%$ de área útil da planta molhada pelo sistema de irrigação, respectivamente) nas subparcelas; e três tipos de coberturas de solo (C1 - mulching preto; C2 - mulching cinza, e C3 - sem cobertura de solo) nas subsubparcelas, com seis repetições, totalizando 12 tratamentos e 72 parcelas experimentais.

O cultivo foi conduzido em espaçamento de $0,30 \mathrm{x}$ 2,00 m (16.666 plantas ha $\left.{ }^{-1}\right)$ em canteiros com $0,25 \mathrm{~m}$ de altura e seis plantas úteis por sub-subparcela.

As adubações foram realizadas conforme recomendação de Cavalcanti et al. (2008) e análise do solo (Tabela 1); inicialmente com aplicação em fundação e no decorrer do ciclo via sistema de irrigação (utilizando-se injetor Venturi). Em fundação foi aplicado o correspondente a 88,89 e $222,22 \mathrm{~kg} \mathrm{ha}^{-1}$ de ureia e superfosfato simples, respectivamente. E em cobertura 177,78 e $66,67 \mathrm{~kg} \mathrm{ha}^{-1}$ de ureia e cloreto de potássio, respectivamente, em 18 aplicações.

O sistema de irrigação constituiu-se de tubos gotejadores (com gotejadores a cada $0,30 \mathrm{~m}$ e vazão de $2,70 \mathrm{~L} \mathrm{~h}^{-1}$ a pressão de serviço de $147 \mathrm{kPa}$ ), instalados próximo às fileiras de planta, com espaçamento de $0,50 \mathrm{~m}$ entre linhas de gotejadores para o tratamento com dois tubos gotejadores por fileira de planta. Os coeficientes de uniformidade de Christiansen e de distribuição, CUC e CUD, foram de 97,83 e $96,27 \%$, respectivamente.

A cobertura do solo com mulching (em polietileno de 25 micras e 1,20 m de largura), foi realizada após instalação e avaliação do sistema de irrigação e da adubação de fundação.

Para o plantio, foi realizado semeio em bandejas plásticas com substrato a base de vermiculita e 10 dias após, as mudas foram transplantadas para o campo experimental. Nos tratamentos com mulching, furos de $0,05 \mathrm{~m}$ de diâmetro foram feitos para colocação das mudas.

Tabela 1 - Características físico-químicas do solo da área experimental.

\begin{tabular}{|c|c|c|c|c|c|c|c|c|c|c|c|c|}
\hline \multirow[t]{2}{*}{ Camada (cm) } & \multirow[t]{2}{*}{$\mathrm{pH}$} & \multirow[t]{2}{*}{$\mathrm{CE}\left(\mathrm{dS} \mathrm{m} \mathrm{m}^{-1}\right)$} & \multirow[t]{2}{*}{$\mathrm{P}_{\text {disp }}\left(\mathrm{mg} \mathrm{dm}^{-3}\right)$} & \multicolumn{6}{|c|}{ Complexo sortivo $\left(\mathrm{cmol}_{\mathrm{c}} \mathrm{dm}^{-3}\right)$} & \multirow[t]{2}{*}{$\operatorname{Dg}\left(\mathrm{g} \mathrm{cm}^{-3}\right)$} & \multirow[t]{2}{*}{ Ucc $(\%$ peso $)$} & \multirow[t]{2}{*}{$\Psi \mathrm{cc}(\mathrm{kPa})$} \\
\hline & & & & $\mathrm{Ca}$ & $\mathrm{Mg}$ & K & $\mathrm{Na}$ & $\mathrm{Al}$ & $\mathrm{H}+\mathrm{Al}$ & & & \\
\hline $0-20$ & 6,72 & 0,58 & 29,64 & 3,61 & 0,58 & 0,49 & 0,04 & 0,00 & ND & 1,85 & 7,65 & 19,95 \\
\hline $20-40$ & 6,87 & 0,64 & 27,85 & 2,90 & 0,42 & 0,44 & 0,04 & 0,00 & ND & 1,66 & 9,13 & 14,95 \\
\hline
\end{tabular}

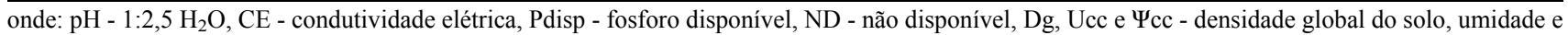
tensão de retenção de água no solo à capacidade de campo, respectivamente (obtidos em testes de campo). Observação: a umidade do solo a ponto de murcha permanente foi considerada como sendo metade da Ucc. 
O manejo da irrigação foi realizado a partir da metodologia de Vermeiren \& Jobling (1980), em função do balanço hídrico sequencial da cultura, com dados de evapotranspiração de referência obtidos de uma estação meteorológica automática, modelo Vantage Pro2, marca Devis, instalada a cerca de $900 \mathrm{~m}$ da área experimental; coeficientes de cultura $(\mathrm{kc})$ de $0,50,1,05$, e 0,75 correspondentes aos kc's inicial, médio e final, respectivamente (Allen et al., 1998) e coeficientes de localização (KL) obtido como a décima parte da raiz quadrada da percentagem de solo molhado (Keller \& Bliesner, 1990), que gerou $\mathrm{KL}$ igual a 0,50 e 0,71 para os tratamentos $\mathrm{S} 1$ e S2, respectivamente.

Para acompanhamento da umidade do solo foram instalados (no $2^{\circ}$ ciclo) tensiômetros de punção nas camadas de 0,00 a 0,20 e de 0,20 a $0,40 \mathrm{~m}$, em três blocos experimentais. A conferência das tensões de retenção de água no solo foi realizada três vezes por semana com uso de tensímetro digital.
Ao final de cada ciclo quantificou-se, em termos comercializáveis (ou comerciais) e totais, o número e a massa de frutos, a produtividade média da cultura e o uso eficiente da água (razão entre a produtividade média da cultura e a lâmina de água aplicada). Para caracterização dos atributos pós-colheita foram realizados, biometria dos frutos (comprimento e largura ou diâmetros longitudinal e transversal), determinação da firmeza, da acidez titulável, do $\mathrm{pH}$ e dos sólidos solúveis totais da polpa dos frutos, a partir da metodologia proposta pelo Instituto Adolfo Lutz (1985).

Os dados foram submetidos à normalidade de resíduos pelo método de Shapiro-Wilk e teste de homocedasticidade de Bartlett, e no caso de significância estatística, as médias foram classificadas segundo teste de Tukey a $5 \%$ de probabilidade, a partir do programa computacional $\mathrm{R}$ versão 3.4.2 (R, 2017), associado ao pacote agricolae (Mendiburu, 2013).

Na Fig. 1 encontram-se os dados meteorológicos registrados durante a pesquisa.

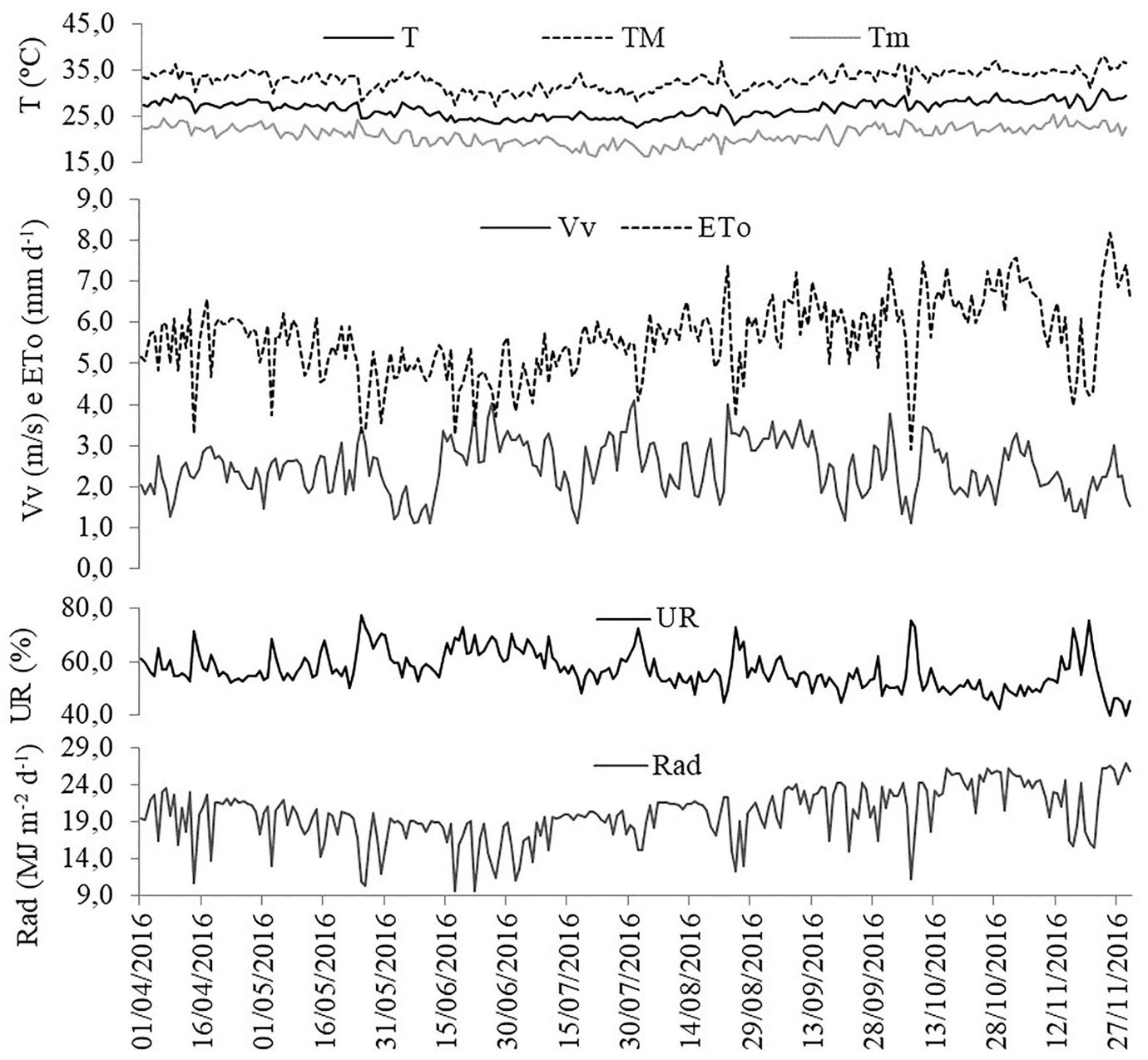

Figura 1 - Dados meteorológicos de temperatura média (T), máxima (TM), mínima (Tm) e umidade relativa (UR) do ar, velocidade do vento (Vv), evapotranspiração de referência (ETo) e radiação solar global (Rad) registradas durante a execução da pesquisa. Petrolina/PE. 


\section{Resultados e Discussão}

As características produtivas dos melões analisados foram estatisticamente equivalentes, com exceção da produtividade comercial no segundo ciclo, em que o 'Gladial' (H1) superou o 'SF 10/00 F1' (H2) em 6,92 $\mathrm{t} \mathrm{ha}^{-1}$ (Tabela 2).

Para os arranjos do sistema de irrigação, o uso de uma fileira de gotejadores por fileira de plantas (S1) propiciou maior número, massa e produtividade comercial dos melões em comparação ao sistema com duas fileiras de gotejadores (S2), no segundo ciclo produtivo. E o uso de mulching independentemente da cor $(\mathrm{C} 1$ e $\mathrm{C} 2)$, ocasionou acréscimos significativos para todas as variáveis avaliadas em ambos os ciclos. Destaque ao incremento de 101,68\% na produtividade comercial dos melões no segundo ciclo para os tratamentos com mulching cinza (C2) em comparado ao cultivo sem mulching, $\mathrm{C} 3$, (Tabela 2 ).

O motivo das melhores respostas do meloeiro ter ocorrido para o arranjo de irrigação com uma fileira de gotejadores por fileira de plantas (S1) pode ter ocorrido devido a menor lâmina de irrigação ser promovida por este tratamento (fator de localização de menor valor) e a cobertura do solo com o mulching reduzir a parcela de água perdida por evaporação, ocasionando menor lixiviação de sais e consequentemente maior disponibilização de nutrientes na zona de absorção radicular efetiva da cultura (Braga et al., 2017), com efeitos positivos na partição e

Tabela 2 - Resumo dos atributos produtivos, número, massa e produtividade de frutos totais e comerciais dos melões 'Gladial' e 'SF 10/00 F1' para dois ciclos produtivos em Petrolina-PE.

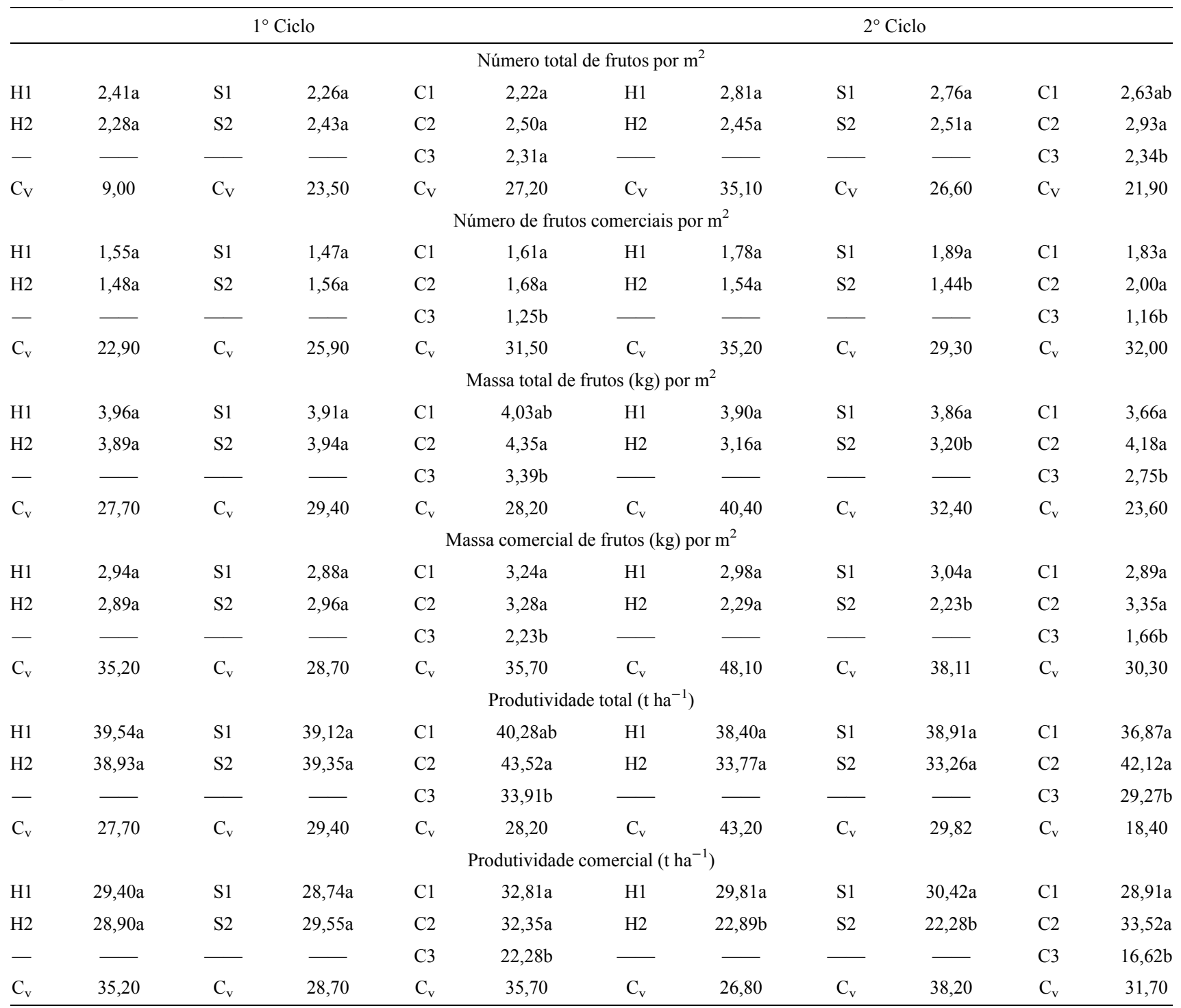

Observação: Médias seguidas das mesmas letras nas colunas não diferem entre si pelo teste de Tukey a 5\% de probabilidade. H1 - melão 'Gladial'; H2 melão 'SF 10/00 F1'; S1 - uma fileira de gotejador; S2 - duas fileiras de gotejadores; C1 - mulching preto, C2 - mulching cinza e C3 - sem mulching. $\mathrm{C}_{\mathrm{V}}$ coeficiente de variação, \%. 
alocação de fotoassimilados para os frutos (Rezzo et al., 2020).

A influência da cobertura do solo já havia sido notada por Câmara et al. (2007) no cultivo do melão amarelo 'Goldex', em Mossoró-RN, onde obtiveram as maiores quantidades de frutos e produtividades totais para os tratamentos com mulching, 21.795,8 frutos ha ${ }^{-1}$ e 35,40 $\mathrm{t} \mathrm{ha}^{-1}$ para densidade de plantio de 20.000 plantas ha ${ }^{-1}$. Neste trabalho, mesmo com menor densidade de plantio, 16.666 plantas ha ${ }^{-1}$, as médias obtidas foram maiores que as dos autores supracitados, o número máximo de frutos chegou aos 29.300 frutos $\mathrm{ha}^{-1}$ (com 20.000 frutos comerciais $\mathrm{ha}^{-1}$ ) e produtividade total de $43,52 \mathrm{t} \mathrm{ha}^{-1}$ para os tratamentos com mulching.

Verificou-se ainda que a produtividade total máxima obtida neste trabalho $\left(43,89 \mathrm{t} \mathrm{ha}^{-1}\right.$, Tabela 2$)$ foi superior as encontradas por Sousa et al. (2010) e Simões et al. (2016) para as cultivares Goldmine e SF 10-00, 34,80 t $\mathrm{ha}^{-1}$ em Fortaleza-CE, e 38,10 $\mathrm{t} \mathrm{ha}^{-1}$ em Petrolina-PE, respectivamente. Mas, levemente superada, em termos de produtividade comercial (máximo de 33,52 $\mathrm{t} \mathrm{ha}^{-1}$, Tabela 2), pela cultivar Araguaia $\left(34,50 \mathrm{t} \mathrm{ha}^{-1}\right.$ de frutos comercias) obtido por Costa et al. (2012) em Petrolina-PE. Injúrias nos frutos podem ter sido os principais motivos do decréscimo produtivo comercial desta pesquisa. Enquanto os refugos, nesta, totalizaram $10,37 \mathrm{t} \mathrm{ha}^{-1}$, no trabalho de Costa et al. (2012) foram registrados apenas 1,50 $\mathrm{t} \mathrm{ha}^{-1}$.

Em relação à quantidade de frutos comerciais por $\mathrm{m}^{2}$, neste trabalho foram verificados, para os tratamentos com uso de mulching, variação de 1,47 a 2,00 (Tabela 2), enquanto que Kosterna et al. (2011) para as cultivares Yupi e Seledyn, obtiveram de 1,39 a 2,36 frutos por $\mathrm{m}^{2}$. Os melões cv. Gladial e SF 10/00 F1 apresentaram ainda maiores valores de massa total e comercial de frutos por $\mathrm{m}^{2}$ (de 3,16 a 3,96 e de 2,29 a $2,98 \mathrm{~kg} \mathrm{~m}^{2}$, respectivamente) do que o melão cv. AF-682, cultivado em JuazeiroBA, 1,62 a 1,83 e 1,44 a $1,61 \mathrm{~kg} \mathrm{~m}^{2}$, respectivamente aos frutos totais e comercias (Batista et al., 2009). Tais diferenças podem estar associadas ao potencial genético dos melões, ao ambiente e ao manejo empregado (Dalastra et al., 2016). Toma-se esta afirmativa como explicação (em termos de variação ambiental meteorológica e edáfica) para as flutuações observadas entre os ciclos produtivos desta pesquisa.

Com relação às variáveis pós-colheita (Tabela 3), observou-se que o comprimento e a largura máxima dos frutos (17,93 e 15,33 cm, respectivamente) foram superiores aos obtidos por Simões et al. (2016), 16,40 e $13,80 \mathrm{~cm}$ (para as variedades SF 10/00 F1 e Goldmine), apresentaram ainda superioridade de mais de $13,27 \%$ à variedade AF-682 do ensaio realizado por Batista et al. (2009), e 36,50\% ao híbrido 'Nectar' da cv. Gália (Lima et al., 2017). Estas características são importantes na classificação dos frutos por tipo, que corresponde à quantidade de melões por caixa padrão de $54 \times 34 \times 17 \mathrm{~cm}$ (Faria et al., 2000). No caso os melões desta pesquisa se enquadraram nos tipos 6 ou 7 (entre 6 e 7 melões por caixa). Tipos estes que já foram os mais indicados para o mercado interno (Faria et al., 2000). No entanto, atualmente os melões tipo 6 e 7 tem igual preferência pelos mercados interno e externo (Dantas et al., 2013). A fonte de adubo nitrogenado adotado nesta pesquisa foi a ureia e isto pode ter sido uma das causas dos frutos terem apresentado comprimento e diâmetro maiores que os registrados na literatura; Rodrigues et al. (2019) constataram aumento de até $175 \%$ na biometria de frutos de melão Cantaloupe quando usada a ureia como fonte de nitrogênio para a planta.

$\mathrm{Na}$ característica firmeza, em ambos os ciclos, os valores obtidos foram inferiores aos alcançados por Câmara et al. (2007) para a cv. Goldex (36,1 a 40,5 N), porém, superiores ao mínimo exigido para o momento da colheita que é de $22 \mathrm{~N}$ (Filgueiras et al., 2000) e aos 23,62 N (2,41 kgf) obtidos por Cavalcante Neto et al. (2020) para o melão 'Gladial'. Considerando-se que este último é um dos híbridos que estão sendo testados, verifica-se que os resultados obtidos neste trabalho se tornam positivos, já que frutos com maior firmeza são mais resistentes às injúrias mecânicas durante o transporte e comercialização, e, geralmente, apresentam maior vida útil póscolheita (Medeiros et al., 2012).

Para a variável acidez titulável, houve efeito significativo para os tratamentos avaliados no primeiro ciclo produtivo (Tabela 3), sendo os resultados obtidos nesse trabalho $(0,99$ a $1,07 \%)$ superiores em até 10 vezes aos encontrados por Yldirim et al. (2009), que estudando um programa de irrigação e requisitos de água apropriados para o melão 'Kırkağaç' em sistema de irrigação por gotejamento em solos com alta capacidade de retenção de água, na Turquia, encontraram valores que variaram de 0,09 a $0,16 \%$.

Em relação aos sólidos solúveis, não foi observado efeito estatístico para as médias dos tratamentos nos dois ciclos produtivos. Contudo, os frutos provenientes do segundo ciclo alcançaram valores de sólidos solúveis próximos e dentro da exigência para comercialização de melões no mercado externo (sólidos solúveis variando de 9,93 a $10,43^{\circ}$ Brix). Esse resultado foi similar ao encontrado por Simões et al. (2016), na ordem de 9,20 a $10,70{ }^{\circ}$ Brix, com as cultivares Goldmine e 10-00 em testes de lâminas de irrigação por gotejamento, na ausência e presença de filme plástico enterrados em diversas profundidades do solo, em Petrolina/PE. Batista et al. (2009) trabalhando com melões irrigados por sulco e gotejamento, no Submédio Vale do São Francisco, obtiveram de 11,09 e 11,15 ${ }^{\circ}$ Brix, valores acimas dos exigidos quando se foca a exportação dos frutos. Para o mercado interno e externo é necessário Brix de no mínimo $10^{\circ}$ (Filgueiras et al., 2000).

Para os tratamentos das cores do mulchings não houve diferença significativa para nenhuma variável (Tabela 3), diferentemente do que foi observado por Gon- 
Tabela 3 - Resumo da classificação de médias para os parâmetros comprimento de frutos, largura de frutos, firmeza, acidez e sólidos solúveis nos dois ciclos produtivos dos melões 'Gladial' e 'SF 10/00 F1' cultivados em Petrolina-PE.

\begin{tabular}{|c|c|c|c|c|c|c|c|c|c|c|c|}
\hline \multicolumn{6}{|c|}{$1^{\circ}$ Ciclo } & \multicolumn{6}{|c|}{$2^{\circ}$ Ciclo } \\
\hline \multicolumn{12}{|c|}{ Comprimento do fruto $(\mathrm{cm})$} \\
\hline H1 & $17,80 \mathrm{a}$ & $\mathrm{S} 1$ & $17,91 \mathrm{a}$ & $\mathrm{C} 1$ & $17,79 \mathrm{a}$ & $\mathrm{H} 1$ & $16,65 \mathrm{a}$ & $\mathrm{S} 1$ & $16,71 \mathrm{a}$ & $\mathrm{C} 1$ & $16,62 \mathrm{a}$ \\
\hline $\mathrm{H} 2$ & $17,45 \mathrm{a}$ & $\mathrm{S} 2$ & $17,33 \mathrm{a}$ & $\mathrm{C} 2$ & $17,14 \mathrm{a}$ & $\mathrm{H} 2$ & $16,92 \mathrm{a}$ & S2 & $16,86 \mathrm{a}$ & $\mathrm{C} 2$ & $16,62 \mathrm{a}$ \\
\hline- & - & - & - & $\mathrm{C} 3$ & $17,93 \mathrm{a}$ & - & - & $\longrightarrow$ & - & $\mathrm{C} 3$ & $17,11 \mathrm{a}$ \\
\hline $\mathrm{C}_{\mathrm{V}}$ & 4,30 & $\mathrm{C}_{\mathrm{V}}$ & 6,10 & $\mathrm{C}_{\mathrm{V}}$ & 7,40 & $\mathrm{C}_{\mathrm{V}}$ & 11,10 & $\mathrm{C}_{\mathrm{V}}$ & 8,20 & $\mathrm{C}_{\mathrm{V}}$ & 11,00 \\
\hline \multicolumn{12}{|c|}{ Largura de frutos $(\mathrm{cm})$} \\
\hline H1 & $15,14 \mathrm{a}$ & $\mathrm{S} 1$ & $15,20 \mathrm{a}$ & $\mathrm{C} 1$ & $15,33 \mathrm{a}$ & $\mathrm{H} 1$ & $13,66 \mathrm{a}$ & $\mathrm{S} 1$ & $13,86 a$ & $\mathrm{C} 1$ & $13,84 \mathrm{a}$ \\
\hline $\mathrm{H} 2$ & $15,13 \mathrm{a}$ & $\mathrm{S} 2$ & $15,07 \mathrm{a}$ & $\mathrm{C} 2$ & $14,96 \mathrm{a}$ & $\mathrm{H} 2$ & $13,98 \mathrm{a}$ & $\mathrm{S} 2$ & $13,78 \mathrm{a}$ & $\mathrm{C} 2$ & $13,63 \mathrm{a}$ \\
\hline- & - & - & - & $\mathrm{C} 3$ & $15,10 \mathrm{a}$ & - & - & 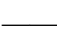 & - & $\mathrm{C} 3$ & $13,99 \mathrm{a}$ \\
\hline $\mathrm{C}_{\mathrm{V}}$ & 10,80 & $\mathrm{C}_{\mathrm{V}}$ & 8,00 & $\mathrm{C}_{\mathrm{V}}$ & 6,00 & $\mathrm{C}_{\mathrm{V}}$ & 3,90 & $\mathrm{C}_{\mathrm{V}}$ & 8,70 & $\mathrm{C}_{\mathrm{V}}$ & 5,10 \\
\hline \multicolumn{12}{|c|}{ Firmeza $(\mathrm{N})$} \\
\hline H1 & $30,13 \mathrm{a}$ & $\mathrm{S} 1$ & $29,06 \mathrm{a}$ & $\mathrm{C} 1$ & $29,26 \mathrm{a}$ & H1 & $30,77 \mathrm{a}$ & $\mathrm{S} 1$ & $29,77 \mathrm{a}$ & $\mathrm{C} 1$ & $27,91 \mathrm{a}$ \\
\hline $\mathrm{H} 2$ & $27,66 \mathrm{a}$ & $\mathrm{S} 2$ & $28,73 \mathrm{a}$ & $\mathrm{C} 2$ & $27,67 \mathrm{a}$ & $\mathrm{H} 2$ & $28,32 \mathrm{a}$ & $\mathrm{S} 2$ & $29,32 \mathrm{a}$ & $\mathrm{C} 2$ & $31,82 a$ \\
\hline- & - & 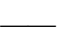 & - & $\mathrm{C} 3$ & $29,75 \mathrm{a}$ & - & - & 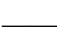 & - & $\mathrm{C} 3$ & $28,91 \mathrm{a}$ \\
\hline $\mathrm{C}_{\mathrm{V}}$ & 25,80 & $\mathrm{C}_{\mathrm{V}}$ & 15,10 & $\mathrm{C}_{\mathrm{V}}$ & 14,20 & $\mathrm{C}_{\mathrm{V}}$ & 36,10 & $\mathrm{C}_{\mathrm{V}}$ & 25,90 & $\mathrm{C}_{\mathrm{V}}$ & 23,90 \\
\hline \multicolumn{12}{|c|}{ Acidez titulável (\% de ácido cítrico) } \\
\hline H1 & $1,05 \mathrm{a}$ & $\mathrm{S} 1$ & $0,99 \mathrm{~b}$ & $\mathrm{C} 1$ & $1,01 \mathrm{a}$ & $\mathrm{H} 1$ & $0,80 \mathrm{a}$ & $\mathrm{S} 1$ & $0,79 \mathrm{a}$ & $\mathrm{C} 1$ & $0,78 \mathrm{a}$ \\
\hline $\mathrm{H} 2$ & $1,00 \mathrm{a}$ & $\mathrm{S} 2$ & $1,07 \mathrm{a}$ & $\mathrm{C} 2$ & $1,05 \mathrm{a}$ & $\mathrm{H} 2$ & $0,76 \mathrm{a}$ & $\mathrm{S} 2$ & $0,78 \mathrm{a}$ & $\mathrm{C} 2$ & $0,80 \mathrm{a}$ \\
\hline- & - & - & - & $\mathrm{C} 3$ & $1,02 \mathrm{a}$ & - & - & $\longrightarrow$ & - & $\mathrm{C} 3$ & $0,76 \mathrm{a}$ \\
\hline $\mathrm{C}_{\mathrm{V}}$ & 19,30 & $\mathrm{C}_{\mathrm{V}}$ & 13,305 & $\mathrm{C}_{\mathrm{V}}$ & 14,80 & $\mathrm{C}_{\mathrm{V}}$ & 24,90 & $\mathrm{C}_{\mathrm{V}}$ & 20,90 & $\mathrm{C}_{\mathrm{V}}$ & 17,90 \\
\hline \multicolumn{12}{|c|}{ Sólidos solúveis ( ${ }^{\circ}$ Brix) } \\
\hline H1 & $8,35 \mathrm{a}$ & $\mathrm{S} 1$ & $8,70 \mathrm{a}$ & $\mathrm{C} 1$ & $8,67 \mathrm{a}$ & $\mathrm{H} 1$ & $9,93 \mathrm{a}$ & $\mathrm{S} 1$ & $10,08 \mathrm{a}$ & $\mathrm{C} 1$ & $10,43 a$ \\
\hline $\mathrm{H} 2$ & $8,84 a$ & S2 & $8,49 \mathrm{a}$ & $\mathrm{C} 2$ & $8,55 \mathrm{a}$ & $\mathrm{H} 2$ & $10,33 \mathrm{a}$ & S2 & $10,17 \mathrm{a}$ & $\mathrm{C} 2$ & $9,96 \mathrm{a}$ \\
\hline- & - & 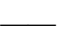 & 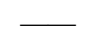 & $\mathrm{C} 3$ & $8,57 \mathrm{a}$ & - & $\longrightarrow$ & 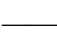 & - & $\mathrm{C} 3$ & $9,99 \mathrm{a}$ \\
\hline $\mathrm{C}_{\mathrm{V}}$ & 17,10 & $\mathrm{C}_{\mathrm{V}}$ & 13,10 & $\mathrm{C}_{\mathrm{V}}$ & 14,00 & $\mathrm{C}_{\mathrm{V}}$ & 14,00 & $\mathrm{C}_{\mathrm{V}}$ & 7,90 & $\mathrm{C}_{\mathrm{V}}$ & 11,80 \\
\hline
\end{tabular}

Observação: Médias seguidas das mesmas letras nas colunas não diferem entre si pelo teste de Tukey a 5\% de probabilidade. H1 - melão 'Gladial'; H2 melão 'SF 10/00 F1'; S1 - uma, e S2 - duas fileira de gotejadores por fileira de plantas; C1 - mulching preto, C2 - mulching cinza e C3 - sem mulching. $\mathrm{C}_{\mathrm{V}}$ - coeficiente de variação, $\%$.

dim et al. (2009), em experimento com híbrido 'Torreon' do grupo Cantaloupe em Mossoró-RN, os quais identificaram que o mulching marrom gerou frutos de melhor qualidade. Neste contexto, cabe relatar a observação de uma menor incidência de mosca branca (Bemisia tabaci), nas fases iniciais do experimento, nos tratamentos com mulching cinza (C2). Isso se deve, certamente, a maior reflexão dos raios solares e irradiância na parte inferior da folha, área esta, de atuação desta praga (Simmons et al., 2010).

No tocante ao acompanhamento da tensão de retenção de água no solo, notou-se similaridade nas duas primeiras fases fenológica para ambos os tratamentos de arranjo de sistemas de irrigação (S1 e S2, uma e duas fileiras de gotejadores por fileira de plantas). Na fase seguinte (floração/frutificação) houve leve superioridade do tratamento $\mathrm{S} 1$, porém, ainda com valores abaixo do valor de referência à capacidade de campo, e na fase de maturação, houve um maior distanciamento das tensões médias do S1 e do S2 (Fig. 2).
O comportamento das tensões de água no solo durante o experimento (Fig. 1) evidencia que os Kc's utilizados nos ensaios foram excessivos para os híbridos estudados e para as formas de cultivo adotadas (cobertura do solo com mulching), propiciando percolação da água e a lixiviação de sais (Bernardo et al., 2019). Isto pode ter gerado prejuízos na produtividade e na qualidade dos frutos da cultura, já que o meloeiro é sensível tanto ao déficit quanto ao excesso hídrico (Pivetta, 2010). Tal hipótese se sustenta na comparação dos dados deste experimento com os valores encontrados por Braga et al., (2017) para as cultivares Tropical e SF 10/00 F1, em Petrolina-PE, cujas produtividades chegaram a $58,20 \mathrm{t} \mathrm{ha}^{-1}$, valor este $33,7 \%$ superior às 43,52 $\mathrm{t} \mathrm{ha}^{-1}$, alcançado neste (Tabela 2).

As maiores tensões foram observadas na fase de floração/frutificação (tensões acima da capacidade de campo) e estas não deve ter sido significativo para proporcionar um déficit hídrico na planta, já que as tensões ficaram próximas ou inferiores a $300 \mathrm{mBar}$, (considerado, segundo Doorenbos \& Pruitt, 1984 e Pires et al., 2013, como tensão 

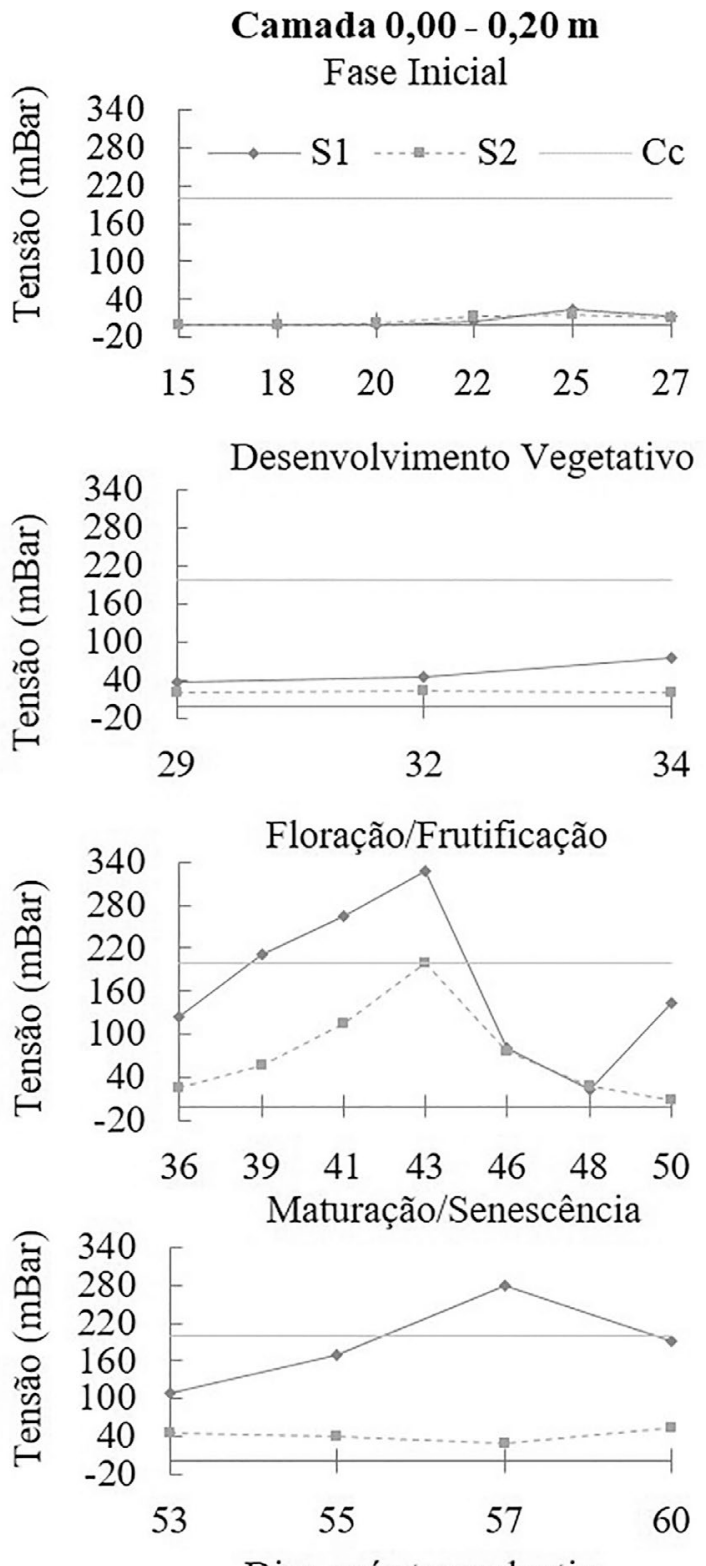

Dias após transplantio
Camada 0,20- 0,40 m

Fase Inicial
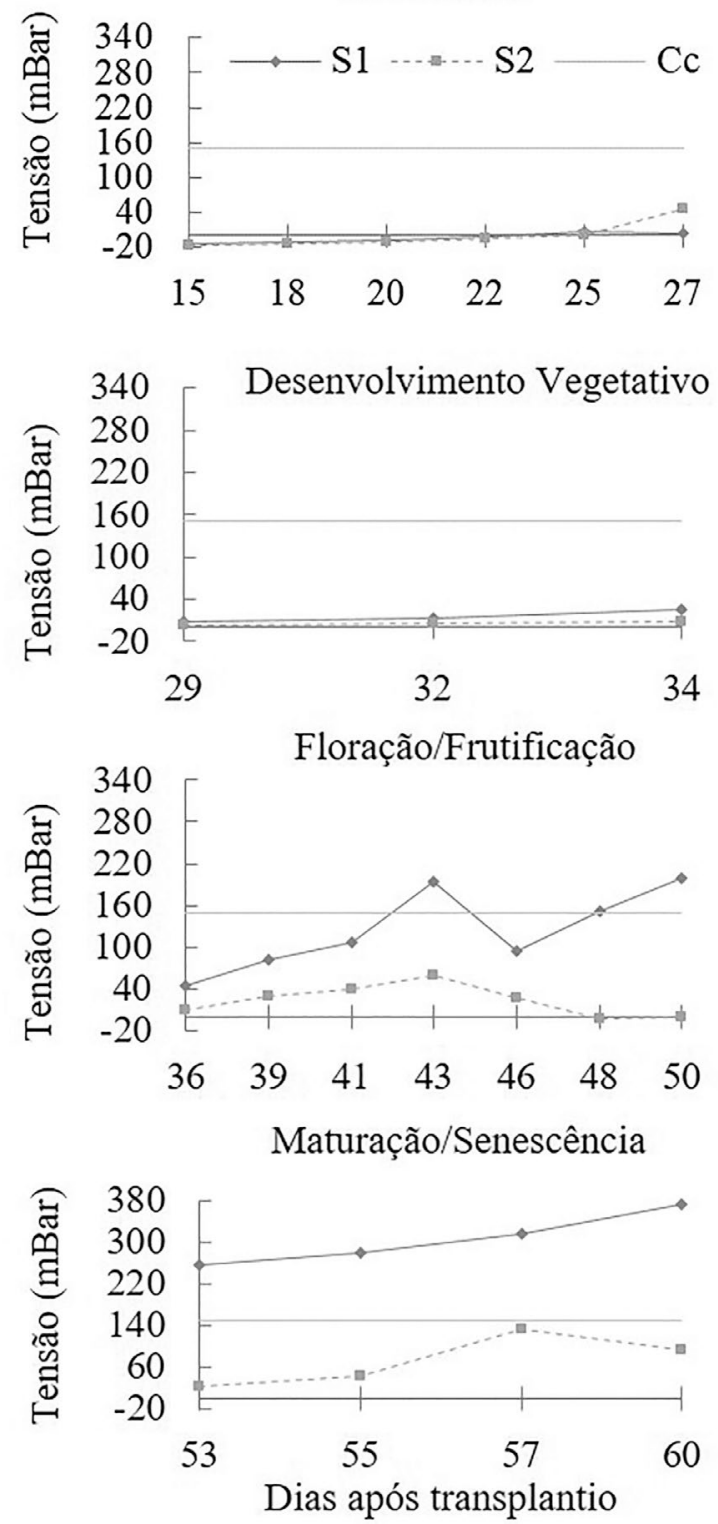

Figura 2 - Comportamento das tensões de água no solo observadas nas diferentes fases fenológicas da cultura, nos dois arranjos dos sistemas de irrigação, com solo coberto por mulching (preto e cinza) e ao longo dos dois ciclos produtivos dos meloeiros nas profundidades de $0,00-0,20$ e $0,20-0,40$ m. Em que: S1 -1 fileira de gotejadores por fileira de plantas; S2 - 2 fileiras de gotejadores por fileira de plantas; Cc - tensão à capacidade de campo. Observações: Tensões adotadas em valores positivos; quanto maior o valor da tensão maior a força de retenção de água no solo e menor a umidade. 1 kPa eqivale a $10 \mathrm{mBar}$.

limite para o meloeiro) e que deficiências dessa magnitude (de até $25 \%$ ) não geraram perdas produtivas em outras variedades de melão (Cabello et al., 2009; Lima et al., 2016). O motivo das maiores tensões terem ocorrido nesta fase fenológica é explicado por Ferreira et al. (1982), Mota et al. (2010) e Pires et al. (2013) que identificaram a floração e início da frutificação como a de maior demanda hídrica do melão, ocasionado pela plena cobertura do solo por folhas e portanto, menor influência do mulching na perda de água para a atmosfera.
Notou-se também que a camada efetiva do sistema radicular da cultura deveria estar compreendida nos primieros 0,20 a $0,30 \mathrm{~m}$ de solo, caracterizado assim pelas maiores tensões registradas na camada mais superifcial do solo em comparação a camada mais subsuperficial, corroborando com Monteiro (2007) que sugere que a camada radicular efetiva do meloeiro varia de 0,30 a $0,50 \mathrm{~cm}$ de profundidade.

As diferenças nas tensões de retenção da água no solo, entre os tratamentos S1 e S2, podem estar associadas 
Tabela 4 - Eficiência no uso da água (EUA, em $\mathrm{kg}$ de melão por $\mathrm{m}^{3}$ de água usada) para dois ciclos produtivos de híbridos de melão amarelos em Petrolina-PE.

\begin{tabular}{lccccccccccc}
\hline \multicolumn{10}{c}{$1^{\circ}$ Ciclo } \\
\hline H1 & $21,20 \mathrm{a}$ & $\mathrm{S} 1$ & $24,49 \mathrm{a}$ & $\mathrm{C} 1$ & $21,60 \mathrm{ab}$ & $\mathrm{H} 1$ & $17,37 \mathrm{a}$ & $\mathrm{S} 1$ & $21,75 \mathrm{a}$ & $\mathrm{C} 1$ & $17,58 \mathrm{a}$ \\
$\mathrm{H} 2$ & $20,77 \mathrm{a}$ & $\mathrm{S} 2$ & $17,48 \mathrm{~b}$ & $\mathrm{C} 2$ & $23,02 \mathrm{a}$ & $\mathrm{H} 2$ & $17,00 \mathrm{a}$ & $\mathrm{S} 2$ & $12,62 \mathrm{~b}$ & $\mathrm{C} 2$ & $20,02 \mathrm{a}$ \\
- & - & - & - & $\mathrm{C} 3$ & $18,32 \mathrm{~b}$ & - & - & - & - & $\mathrm{C} 3$ & $13,95 \mathrm{~b}$ \\
$\mathrm{C}_{\mathrm{V}}$ & 30,30 & $\mathrm{C}_{\mathrm{V}}$ & 29,93 & $\mathrm{C}_{\mathrm{V}}$ & 28,62 & $\mathrm{C}_{\mathrm{V}}$ & 39,34 & $\mathrm{C}_{\mathrm{V}}$ & 38,17 & $\mathrm{C}_{\mathrm{V}}$ & 21,47 \\
\hline
\end{tabular}

Obs.: Médias seguidas das mesmas letras nas colunas não diferem entre si pelo teste de Tukey a 5\% de probabilidade. H1 - melão 'Gladial'; H2 - melão 'SF 10/00 F1'; S1 - uma fileira de gotejador; S2 - duas fileiras de gotejadores; C1 - mulching preto, C2 - mulching cinza e C3 - Sem mulching. $\mathrm{C}_{\mathrm{V}}$ - coeficiente de variação, \%.

à maior quantidade de água aplicada no arranjo do sistema de irrigação com duas fileiras de gotejadores por fileira de plantas (S2). Como o fator de localização (KL) adotado neste trabalho foi obtido a partir da equação de Keller e Bliesner (1990), gerando KL de 0,50 e 0,71 para os tratamentos S1 e S2, respectivamente, ou seja, maiores lâminas para o tratamento $\mathrm{S} 2$, e como ocorre redução da evaporação da água do solo coberto com o mulching (Negreiros et al., 2005), houve maior excedente hídrico no tratamento S2, fato evidenciado na Fig. 2, e que explica a ocorrência de $8,14 \mathrm{t} \mathrm{ha}^{-1}$ a mais de produtividade comercial para o tratamento S1 (Tabela 2), e consequentemente, maior eficiência no uso da água, até $9,13 \mathrm{~kg} \mathrm{~m}^{-3}$ a mais em comparação ao tratamento $\mathrm{S} 2$, no $2^{\circ}$ ciclo (Tabela 4).

Os maiores valores de EUA médios obtidos neste trabalho, 24,49 e $20,38 \mathrm{~kg} \mathrm{~m}^{-3}$, ou 41 e $49 \mathrm{~L}$ de água por $\mathrm{kg}$ de melão, respectivamente para $\mathrm{o} 1^{\circ}$ e $2^{\circ}$ ciclo produtivo, foram superiores aos encontrados por Cardoso (2002) e Batista et al. (2009), que atingiram, respectivamente, 19,14 e $17,72 \mathrm{~kg} \mathrm{~m}^{-3}$, com os melões rendilhado e amarelo em Piracicaba/SP e Petrolina-PE. Já em comparação com o trabalho de Braga et al. (2017) os resultados foram similares, no qual os autores avaliaram as cultivares $\mathrm{SF}$ 10/00 F1 e Tropical em Petrolina/PE, e obtiveram $1 \mathrm{~kg}$ de melão para cada $46 \mathrm{~L}$ de água usada. A EUA é um indicativo do nível de adequação do manejo aplicado ou atingido por uma dada cultura. Quando usado para comparação, expressa qual situação se sobressai, sendo esta a de maior valor numérico (Cardoso, 2002). No entanto, a conotação econômica e ambiental é que definirá a maior ou menor importância deste parâmetro no ato comparativo. Nas regiões áridas e semiáridas, por exemplo, qualquer economia de água pode resultar em sucesso e perpetuação do empreendimento agrícola (Paz et al., 2000; Paz et al., 2002; Carvalho et al., 2015). Neste trabalho, a utilização dos híbridos 'Gladial' e 'SF 10-00 F1' irrigado com uma fileira de gotejadores por fileira de plantas (proporcionalmente de menor custo aquisitivo se comparado ao sistema com duas fileiras de gotejadores por fileira de plantas) e com cobertura do solo com mulching (preto ou cinza), para densidade de 16.667 plantas por hectare, configuraram melhor situação do que as obtidas por Cardoso (2002), Batista et al. (2009) e Braga et al. (2017).

\section{Conclusão}

Os híbridos de melão amarelo 'Gladial' e 'SF 10/00 F1' apresentam semelhantes potenciais produtivos e de qualidade em cultivo com solo coberto por mulching, cinza ou preto, e sistema de irrigação com uma ou duas fileiras de gotejadores por fileira de plantas;

A massa, a quantidade e a produtividade comerciais de frutos de melão 'Gladial' e 'SF 10/00 F1' foram superiores com o uso do mulching.

O uso de mais de uma linha de gotejadores por fileira de plantas provocou maiores desperdícios de água em cultivos com solo coberto por mulching.

A maior economia do uso da água no cultivo dos meloeiros testados ocorreu com o solo coberto por mulching e irrigado por uma fileira de gotejadores por fileira de plantas.

\section{Agradecimentos}

Os autores agradecem ao IF Sertão-PE pelo custeio de bolsas de iniciação científica, insumos de campo e laboratoriais, bem como cedência de espaço físico para a condução da pesquisa. Agradecem também a Embrapa Semiárido pelo empréstimo de equipamentos e apoio técnico.

\section{Referências}

ALLEN, R.G.; PEREIRA, L.S.; RAES, D.; SMITH, M. Crop Evapotranspiration: Guidelines for Computing Crop Water Requirements. Irrigation and Drainage Paper 56. Rome: FAO, 297 p., 1998.

AZEVEDO, P.V.; SILVA, B.B.; SILVA, V.P.R. Walter requirements of irrigated mango orchards in Northeast Brazil. Agricultural Water Management, v. 58, n. 3, p. 241-245, 2003.

BARROS, V.S.; SANTOS, T.L.; SILVA, E.O.; SOUSA, J.A.; FIGUEIRÊDO, M.C.B. Agronomic and environmental performance of melon produced in the brazilian semiarid region. Revista Caatinga, v. 32, n. 4, p. 877-888, 2019.

BATISTA, P.F.; PIRES, M.M.M.L.; SANTOS,J.S.; QUEIROZ, S.O.P.; ARAGÃO, C.A.; DANTAS, B.F. Produção e qualidade de frutos de melão submetidos a dois sistemas de irri- 
gação. Horticultura Brasileira, v. 27, n. 2, p. 246-250, 2009.

BERNARDO, S.; MANTOVANI, E.C.; SILVA, D.D.; SOARES, A.A. Manual de Irrigação. 9. ed. Viçosa: UFV, 545 p., 2019.

BRAGA, M.B.; MAROUELLI, W.A.; RESENDE, G.M.; MOURA, M.S.B.; COSTA, N.D.; CALGARO, M.; CORREIA, J.S. 2017. Coberturas do solo e uso de manta agrotêxtil (TNT) no cultivo do meloeiro. Horticultura Brasileira, v. 35, n. 1, p. 147-153, 2017.

CABELLO, M.J.; CASTELLANOS, M.T.; ROMOJARO, F.; MARTINEZ-MADRID, C.; RIBAS, F. Yield and quality of melon grown under different irrigation and nitrogen rates. Agricultural Water Management, v. 96, n. 5, p. 866-874, 2009.

CÂMARA, M.J.T.; NEGREIROS, M.Z.; MEDEIROS, J.F.; BEZERRA NETO, F.; BARROS JÚNIOR, A.P. Produção e qualidade de melão amarelo influenciado por coberturas do solo e lâminas de irrigação no período chuvoso. Ciência Rural, v. 37, n. 1, p. 58-63, 2007.

CARDOSO, S.S. Dose de CO2 e de Potássio Aplicados Através de Irrigação no Meloeiro Rendilhado (Cucumis melo L.) Cultivado em Ambiente Protegido. Tese de Doutorado, Escola Superior de Agricultura Luiz de Queiroz. Universidade de São Paulo, 2002.

CARVALHO, C.M.; MARINHO, A.B.; VIANA, T.V.A.; JÚNIOR, M.V.; FILHO, R.R.G.; CARVALHO, L.L.S. Eficiência do uso da água na produção do pinhão-manso no semiárido nordestino. Revista Agrarian, v. 8, n. 29, p. 296-303, 2015.

CAVALCANTE NETO, J.G.; FERREIRA, K.T.C.; ARAGÃO, F.A.S.; ANTÔNIO, R.P.; NUNES, G.H.S. Potential of parents and hybrids experimental of the yellow melon. Ciência Rural, v. 50, n. 2, p. 1-9, 2020.

CAVALCANTI, F.J.A.; LIMA JÚNIOR, M.A.; LIMA, J.F.W.F. Recomendações de Adubação para o Estado de Pernambuco: $2^{\text {a }}$ Aproximação. 3 ed. Recife: Instituto Agronômico de Pernambuco, 212 p., 2008.

COSTA, N.D.; RESENDE, G.M. DE.; YURI, J.E.; PETRERE, V.G.; PINTO, J.M.; FERREIRA, T.S.D. Produtividade e qualidade de frutos de melão em dois métodos de irrigação no Submédio São Francisco. Horticultura Brasileira, v. 30, n. 2, p. 2605-2611, 2012.

DALASTRA, G.M.; ECHER, M.M.; KLOSOWSKI, É.S.; HACHMANN, T.L. Produção e qualidade de três tipos de melão, variando o número de frutos por planta. Revista Ceres, v. 63, n. 4, p. 523-531, 2016.

DANTAS, I.C.; OLIVEIRA, C.W.; SILVA, F.L.; SANTOS, F.S.S.; MARCO, C.A. Produção de melão amarelo sob diferentes densidades de plantio. Revista Brasileira de Agricultura Irrigada, v. 7, n. 1, p. 74-84, 2013.

DOORENBOS, J.; PRUITT, W.O. Las Necesidades de Agua de Los Cultivos. Riego y Drenaje, Piper 24, Roma: FAO, 194 p., 1984.

EMBRAPA, CENTRO NACIONAL DE PESQUISA DE SOLOS. Sistema Brasileiro de Classificação de Solos. 2. ed. Rio de janeiro: Embrapa, 101 p., 2006.

FARIA, C.M.B.; COSTA, N.D.; PINTO, J.M.; BRITO, L.T.L.; SOARES, J.M. Níveis de nitrogênio por fertirrigação e densidade de plantio na cultura do melão em um vertissolo. Pesquisa Agropecuária Brasileira, v. 35, n. 3, p. 491-495, 2000.

FERREIRA, F.A.; PEDROSA, J.F.; ALVARENGA, M.A. Melão: cultivares e métodos culturais. Informe Agropecuário, v. 8, n. 85, p. 26-28, 1982.

FILGUEIRAS, H.A.C.; MENEZES, J.B.; ALVES, R.E.; COSTA, F.V.; PEREIRA, L.S.E.; GOMES JUNIOR, J. Colheita e manuseio pós-colheita. In: ALVES, R.E. (coord.), Melão Pós-Colheita. Fortaleza: Embrapa Agroindústria Tropical, p. 23-43, 2000.

GONDIM, A.R.O.; NEGREIROS, M.Z.; MEDEIROS, J.F.; PORTO, D.R.Q.; ALMEIDA-NETO, A. J.; MENEZES, J. B. Qualidade de melão 'Torreon' cultivado com diferentes coberturas de solo e lâminas de irrigação. Revista Ceres, v. 56, n. 3, p. 326-331, 2009.

GUIMARÃES, B.R.; ARAUJO, A.R.R.; GALVÃO, J.R.; PACHECO, M.J.B.; SILVA, S.B.; ASSIS, L.F.C.T.; AZEVEDO, J.C.; MORAES, K.C. Melão (Cucumis melo L.): interrelações entre adubação, nutrição mineral e produção. Revista Ibero-Americana de Ciências Ambientais, v. 11, n. 1, p. 391-399, 2020.

IBARRA, L.; FLORES, J.; DÍAZ-PEREZ, J.C. Growth and yield musk-melon in response to plastic mulch and row covers. Scientia Horticulture, v. 87, n. 1, p. 139-145, 2001.

INSTITUTO ADOLFO LUTZ. Normas Analíticas, Métodos Químicos e Físicos para Análise de Alimentos. 3. ed. São Paulo: Instituto Adolfo Lutz, v. 1, 533 p., 1985.

KELLER, J.; BLIESNER, R.D. Sprinkle and Trickle Irrigation. New York: Van Nostrand Reinold, 652 p., 1990.

KOSTERNA, E.; ZANIEWICZ-BAJIKOWSKA, A.; FRANCZUK, J.; ROSA, R.; CHROMINSKA, K.; BORYSIAKMARCINIAK, I.; PANASZ, M. Effect of synthetic mulches on melon (Cucumis melo L.) yielding. Folia Horticulturae, v. 23, n. 2, p. 151-156, 2011.

LIMA, E.M.C. Irrigação do Meloeiro Cultivado em Ambiente Protegido. Tese de Doutorado, Universidade Federal de Lavras, 2015.

LIMA, E.M.C.; CARVALHO, J.A.; VIOL, M.A.; ALMEIDA, R.C.; REZENDE, F.C. Gália melons production in protected environment under different irrigation depths. Engenharia Agrícola, v. 37, n. 1, p. 75-83, 2017.

LIMA, E.M.C.; CARVALHO, J.A.; VIOL, M.A.; REZENDE, F.C.; GOMES, L.A.A. Resposta do melão tipo Gália a diferentes tensões de água no solo. Revista Brasileira de Agricultura Irrigada, v.10, n. 5, p. 904-913, 2016.

MEDEIROS, J.F.; AROUCHA E.M.M.; DUTRA, I.; CHAVES, S.W.P.; SOUZA, M.S. Efeito da lâmina de irrigação na conservação pós-colheita de melão Pele de Sapo. Horticultura Brasileira, v. 30, n. 3, p. 514-519, 2012.

MEDEIROS, J.F.; SILVA, M.C.C.; CÂMARA NETO, F.G.; ALMEIDA, A.H.B.; SOUZA, J.O.; SOUZA, J.O.; NEGREIROS, M.Z.; SOARES, S.P.F. Crescimento e produção do melão cultivado sob cobertura de solo e diferentes freqüências de irrigação. Revista Brasileira de Engenharia Agrícola e Ambiental, v.10, n. 4, p. 792-797, 2006.

MENDIBURU, F. Statistical Procedures for Agricultural Research. Package 'Agricolae,' version 1.4-4. Comprehensive R Archive Network, Institute for Statistics and 
Mathematics. Disponível em http://cran.r-project.org/web/ packages/agricolae/agricolae.pdf, acesso em 5 de nov. 2013.

MONTEIRO, R.O.C. Influência do Gotejamento Subterrâneo e do 'Mulching' Plástico na Cultura do Melão em Ambiente Protegido. Tese de Doutorado, Escola Superior de Agricultura Luiz de Queiroz. Universidade de São Paulo, 2007.

MOTA, J.C.A.; LIBARDI, P.L.; BRITO, A.S.; ASSIS JÚNIOR, R.N.; AMARO FILHO, J. Armazenagem de água e produtividade de meloeiro irrigado por gotejamento, com a superfície do solo coberta e desnuda. Revista Brasileira de Ciência do Solo, v. 34, n. 5, p. 1721-1731, 2010.

NEGREIROS, M.Z.; COSTAI, F.A.; MEDEIROS, J.F.; LEITÃO, M.M.V.B.R.; BEZERRA NETO, F.; ESPÍNOLA SOBRINHO, J. Rendimento e qualidade do melão sob lâminas de irrigação e cobertura do solo com filmes de polietileno de diferentes cores. Horticultura Brasileira, v. 23, n. 3, p. 773-779, 2005.

PAZ, V.P.S.; TEODORO, R.E.F.; MENDONCA, F.C. Recursos hídricos, agricultura irrigada e meio ambiente. Revista Brasileira de Engenharia Agrícola e Ambiental, v. 4, n. 3, p. 465-473, 2000.

PAZ, V.P.S.; FRIZZONE, J.A.; BOTREL, T.A.; FOLEGATTI, M.V. Otimização do uso da água em sistemas de irrigação por aspersão. Revista Brasileira de Engenharia Agrícola e Ambiental, v. 6, n. 3, p. 404-408, 2002.

PEREIRA, F.A.L.; MEDEIROS, J.F.; GHEYI, H.R.; DIAS, N.S.; PRESTON,W.; VASCONCELOS, C.B.L. Tolerance of melon cultivars to irrigation water salinity. Revista Brasileira de Engenharia Agrícola e Ambiental, v. 21, n. 12, p. $846-851,2017$.

PINTO, M.M.F.; GONÇALVES, J.S.; SOUZA, I.T.N.; BATISTA, N.V.; MELO, V.L.L.; FIRMINO, S.S.; PINEDO, L.Á.; LIMA, P.O. Utilização do melão (Cucumis melo L.) na alimentação de ruminantes: Uma revisão. Brazilian Journal of Development, v. 5, n. 12, p. 31466-31481, 2019.

PIRES, M.M.M.L.; SANTOS, H.S.; SANTOS, D.F.; VASCONCELOS, A.S.; ARAGÃO, C.A. Produção do meloeiro submetido a diferentes manejos de água com o uso de manta de tecido não tecido. Horticultura Brasileira, v. 31, n. 2, p. 304-310, 2013.

PIVETTA, C.R. Posição dos Gotejadores e Cobertura do Solo com Plástico, Crescimento Radicular, Produtividade e
Qualidade do Melão. Tese de Doutorado, Universidade Federal de Santa Maria, 2010.

R Foundation. The R Foundation for Statistical Computing Platform. R Version 3.4.2. Disponível em http://www.r-pro ject.org, acesso em 28 de set. 2017.

REZZO, D.D.P.Z.; SILVA, H.L.O.; SOUSA, F.F.; CAETANO, E.J.M.; QUEIROGA, R.C.F.; SILVA, L.J.S.; SILVA, F.A. Fitomassa e produtividade da melancieira cultivada sob diferentes números de frutos e espaçamento de plantio. Research, Society and Development, v. 9, n. 8, p. 1-16, 2020.

RODRIGUES, E.N.S.; SOUSA, F.A.R.M.; SILVA, S.M.; SANTOS, K.M.; MENDONÇA, R.M.N.; SILVA, P.C.A. Influência de fontes de nitrogênio na biometria do melão Cantaloupe 'Hy Mark' sob as condições do Brejo Paraibano (Brasil). Revista Brasileira de Meio Ambiente, v. 7, n. 3, p. 48-57, 2019.

SILVA, L.T.; SILVA, E.O.; FIGUEIRÊDO, M.C.B.; CORRÊA, L.C.; ARAGÃO, F.A.S. Pós-colheita do melão amarelo 'Goldex' cultivado sob adubação verde e plantio direto com diferentes coberturas. Irriga, v. 21, n. 4, p. 764-778, 2016.

SIMMONS, A.M.; KOUSIK, C.S.; LEVI, A. Combining reflective mulch and host plant resistance for sweetpotato whitefly (Hemiptera: Aleyrodidae) management in watermelon. Crop Protection, v. 29, n. 8, p. 898-902, 2010.

SIMÕES, W.L.; ANJOS, J.B.; COELHO, D.S.; YURI, J.E.; COSTA, N.D.; LIMA, J.A. Uso de filmes plásticos no solo para o cultivo de meloeiro irrigado. Water Resources and Irrigation Management, v. 5, n. 1, p. 23-29, 2016.

SOUSA, A.E.C.; BEZERRA, F.M.L.; SOUSA, C.H.C.; SANTOS, F.S.S. Produtividade do meloeiro sob lâmina de irrigação e adubação potássica. Engenharia Agrícola, v. 30, n. 2, p. 271-278, 2010.

VERMEIREN, L.; JOBLING, G.A. Localized Irrigation. Irrigation and Drainage, Paper 36. Rome: FAO, 203 p., 1980.

YILDIRIM, O.; HALLORAN, N.; ÇAVUSOGLU, S.; SENGÜL, N. Effects of different irrigation programs on the growth, yield, and fruit quality of drip-irrigated melon. Turkish Journal of Agriculture and Forestry, v. 33, n. 3, p. 243-255, 2009.

License information: This is an open-access article distributed under the terms of the Creative Commons Attribution License (type CC-BY), which permits unrestricted use, distribution and reproduction in any medium, provided the original article is properly cited. 